\title{
Effectiveness of Coping Strategies Program in Reducing the Rejection Sensitivity and Social Anxiety with College Students
}

\author{
Elballah A. Khaled \\ Department of Special Education, College of Education, King Faisal University, KSA
}

Received November 21, 2020; Revised April 2, 2021; Accepted April 18, 2021

\section{Cite This Paper in the following Citation Styles}

(a): [1] Elballah A. Khaled, "Effectiveness of Coping Strategies Program in Reducing the Rejection Sensitivity and Social Anxiety with College Students," Universal Journal of Educational Research, Vol. 9, No. 5, pp. 901 - 910, 2021. DOI: 10.13189/ujer.2021.090502.

(b): Elballah A. Khaled (2021). Effectiveness of Coping Strategies Program in Reducing the Rejection Sensitivity and Social Anxiety with College Students. Universal Journal of Educational Research, 9(5), 901 - 910. DOI: 10.13189/ujer.2021.090502.

Copyright $\bigcirc 2021$ by authors, all rights reserved. Authors agree that this article remains permanently open access under the terms of the Creative Commons Attribution License 4.0 International License

\begin{abstract}
Coping strategies may play a central role in reducing rejection sensitivity and social anxiety, so an examination of coping strategies is important. Rejection sensitivity and social anxiety are undoubtedly parts of human psychology. Some types of behaviors, such as avoiding performing in groups and withdrawal due to rejection sensitivity, may indicate that students have difficulties coping with social situations. Therefore, this study aims to examine the improvement of coping strategies and their effect on reducing rejection sensitivity and social anxiety. The study will also determine the differences between the two coping strategies in college students. Participants in this study consisted of 60 students aged 19-22 years from the King Faisal University College of Education. The experimental group of 20 students, who showed higher levels of rejection sensitivity and social anxiety, responded to the coping strategies scale, rejection sensitivity scale, and social anxiety scale for college students. This study used a t-test and the Mann Whitney test to determine the differences between pre- and post-test results. We applied a counseling program which consisted of 16 sessions, focusing on improving productive coping strategies in the areas of cognitive restriction, expressing emotions, social support, and reducing nonproductive coping strategies such as wishful thinking, self-criticism, and social withdrawal. The results showed statistically significant differences in coping strategies between pre- and post-test scores in the experimental group
\end{abstract}

in favor of the posttest. In addition, there are significant differences in rejection sensitivity and social anxiety between pre- and posttest results in favor of the post-test. Our findings highlight the importance of productive coping strategies in reducing rejection sensitivity and social anxiety in the sample. The findings of this study can contribute to the improvement of college counseling programs involving coping strategies. In summary, the findings suggest that high levels of productive coping strategies can contribute to the reduction of rejection sensitivity and social anxiety, especially in adolescents.

Keywords Coping Strategies, Rejection Sensitivity, Social Anxiety, College Students

\section{Introduction}

Adolescence is a period in life with many psychological, cognitive, social changes combined with diverse sources of stress. When the students separate from their peer group after secondary school to pursue study at a university, which is a larger world with a different system than their previous school, they lose friends and peers, many of whom choose to attend different colleges. Consequently, students often face isolation, loneliness, rejection sensitivity, and social anxiety. Students use 
cognitive and behavioral coping strategies to adapt to these new challenges, which have effects on social and emotional development. Entering college requires youths to face multiple transitions, including changes in living arrangements, academic environments, and social relationship networks [29]. Adolescence is recognized as a particularly stressful period of development. During this period, adolescents simultaneously deal with physical and cognitive transitions as well as the challenges of changing peer relationships. Few studies on coping strategies with adolescents focus on developmental changes in coping behaviors or on how coping strategies may vary with gender and types of stressors [35]. According to Lazarus and Folkman [21] coping involves "cognitive, behavioral and emotional efforts to manage particular external and/or internal demands that are appraised as taxing or exceeding the resources of the person." According to Lopze [23], coping is "a response aimed at diminishing the physical, emotional, and psychological burden that is linked to stressful life events and daily hassles." The conceptualization of coping processes has evolved over the past several decades resulting in several contemporary models of coping. One of the widely accepted models is known as the transactional model of stress appraisal and coping developed by Lazarus and Folkman.

The use of avoidance-oriented strategies is generally associated with adolescents' poor adaptation and high levels of problem behaviors. Avoidance-oriented coping often exacerbates stress because it fails to help individuals deal with the things that make them stressed. Instead, it includes denying that the stressful event or its impact occurred, thus physically removing oneself from the situation by escaping [15]. The use of emotion-oriented coping seems to be related to negative outcomes. Some studies report that it is associated with internalizing problems, such as depression and anxiety. Emotion-focused coping may lead to an intensification of emotional distress, partially because individuals are more focused on the emotional experience rather than finding problem-solving strategies to deal with stressful events. Richardson [30] investigated assessing coping (i.e., avoidant, problem-solving, social-support-seeking) in light of symptoms of psychopathology (i.e., generalized anxiety, social anxiety, depression, eating pathology). The study found that avoidant coping predicted increases in symptoms of social anxiety, and depressive symptoms predicted increases in maladaptive coping. There is evidence for an association between problem-solving and symptoms of anxiety, and symptoms of depression predict reduced problem-solving coping and social-support-seeking from friends over time.

McDonald [26] examined the relationship between rejection sensitivity, relationships with parents and friends, and internalizing symptoms during adolescence. One particular interest was whether the quality of relationships with mothers, fathers, and friends would moderate associations between rejection sensitivity and depressive symptoms and social anxiety and between rejection sensitivity and social anxiety. London et al. [24] confirmed the idea that anxious expectations of rejection can lead to withdrawal and social anxiety. The study also found that teachers with positive attitudes can help students with high rejection sensitivity.

As can be seen from the model, the most important part of the rejection sensitivity dynamics is the anxious anticipation of rejection. Both this rejection and having a high level of anxiety about what could happen is described as high rejection sensitivity, while students with low rejection sensitivity do not feel anxious about whether they will be accepted or rejected [9]. In this context, Khoshkam et al. [20] found that anxiety levels and self-confidence in university students were partial tool variables in the relationship between anxiety-related attachment styles and rejection sensitivity. Rejection sensitivity seems to show a significant positive correlation to fearful and preoccupied attachment styles and worry, and a significant negative correlation to self-esteem. Similarly, Ozen et al. [28] found that attachment anxiety and avoidance, as well as rejection sensitivity, had neither unique nor mediated effects on friendship quality. However, rejection sensitivity moderated the effect of attachment avoidance on friendship quality, and the combination of high avoidance and high rejection sensitivity is associated with particularly low friendship quality.

Nevertheless, Downey et al. [10] argued that there may be different correlates and consequences of rejection sensitivity anxious and rejection sensitivity angry forms. They asserted that rejection sensitivity anxiety would be more strongly associated with internalizing problems, such as depressive affect and social anxiety, whereas rejection sensitivity angry would be more strongly associated with externalizing problems, such as aggressive behavior.

Many researchers have suggested that interpersonal experiences affect individuals' expectations about future relationships. This is especially true for individuals who have a history of expectations of rejection and anxiety from others in social situations as opposed to those who feel satisfaction in their relationships. According to previous studies (e.g., [6,7]), proactive coping strategies may be more effective through counseling programs with adolescents, especially with older adolescents, because they have broader experience, higher intellectual ability, and greater general competence than younger adolescents. Expectations of social anxiety are higher with college students who have high rejection expectations as compared to peers with lower social anxiety. Therefore, stressors in social situations are associated with rejection expectations in relationships in general. The study may guide students to develop suitable coping strategies to reduce rejection sensitivity and social anxiety.

\subsection{Statement of the Problem}

Downey \& Feldman [9] found that rejection sensitivity 
may lead people to be unable to maintain supportive and satisfying relationships because they readily avoid situations in which rejection is possible. Downey and Feldman's model suggests that people who enter a relationship anxiously expecting rejection are likely to perceive intentional rejection as a result of their partner's insensitive or ambiguous behaviors, feel insecure and unhappy about the relationship, and respond to perceived rejection or threats of rejection by their partner with hostility, diminished support, jealousy, or behavior control. From the point of view of this theory, we can conclude that people with high rejection sensitivity are not only unable to maintain supportive relationships, but due to their lack of quality relationships, may also perceive less social support from those relationships they do have [18].

Al-Hazmi [2] aimed to assess the prevalence and associated factors of social anxiety disorder and its effect on the performance of medical students at Taibah University in Medina. He found that $13.5 \%$ of the students in the study reported severe to very severe social anxiety disorder, $18.8 \%$ reported mild level of the disorder, and $19.6 \%$ reported moderate level.

In another study in Saudi Arabia, Hakami [14] found that $47.2 \%$ of university students had mild symptoms of social anxiety, $42.3 \%$ had moderate to marked symptoms, and $10.5 \%$ had severe to very severe symptoms of social anxiety disorder.

Previous studies have shown that more than half (65.4\%) of university students had moderate social anxiety, while nearly a quarter $(22.4 \%)$ had severe levels of the disorder. Social anxiety not only seriously endangers the psychosocial adaptation, interpersonal communication, and academic achievement of college students but also leads to internalization and externalization problems such as depression and aggressive behaviors [5, 27, 37, 38].

The researcher noted that many college students had a feeling of rejection sensitivity and social anxiety, because they were not using proactive coping strategies, resulting in withdrawal from communication and interaction situations. Consequently, this research attempts to answer the following questions:

1. How effective are counseling programs effective in improving coping strategies, and what are their effects on reducing rejection sensitivity and social anxiety in college students?

2. Are there differences between rankings of mean scores in the experimental group in coping strategies, rejection sensitivity, and social anxiety pre- and posttest?

\subsection{Significance of the Research}

The theoretical and practical importance of the current research is determined as follows:

A- Theoretical importance:
Despite the previous studies that dealt with coping strategies and their impact on human behavior, studies have not given sufficient attention to counseling programs that enhance the dimensions of coping strategies, whether based on emotion or problems.

- The current study deals with important problems from which some university students suffer, such as rejection sensitivity and social anxiety, which affect their levels of social and academic progress. although they are in the preparation stage to work as teachers, and this work needs to enhance the skills of social interaction.

\section{B- Practical importance:}

- Preparing a counseling program to improve the dimensions of coping strategies among university students.

- Presenting new psychological scales for the Arab library, such as a coping strategies scale, a rejection sensitivity scale, and a social anxiety scale.

- Helping educational and psychological researchers recognize coping strategies that can be used by students to reduce rejection sensitivity and social anxiety.

- Training students by educators to enhance effective coping strategies in interaction situations, with the aim of reducing rejection sensitivity and social anxiety.

- Using techniques as an intervention method for university students with high rejection sensitivity and social anxiety .

\subsection{Research Objective}

The current study aims to:

1. Verify the effectiveness of counseling programs in improving coping strategies and their effect on reducing rejection sensitivity and social anxiety in college students.

2. Determine differences in coping strategies, rejection sensitivity, and social anxiety in college students.

\subsection{Definitions of the Main Concepts}

Coping strategies: Coping is defined as cognitive and behavioral efforts used to manage demands of stressful situations as well as emotions surrounding those situations [21]. According to Lewis \& Frydenberg [22], coping strategies are "behavioral and cognitive efforts used by individuals to manage the demands of a personenvironment relationship." For this study, we defined coping strategies as the total score obtained by students on the scale of coping strategies prepared by Tobin [34].

Rejection sensitivity: Rejection sensitivity is defined as "sensitivity to ostracism" as a "dispositional" mountainous predisposition and represents an increase in the tendency 
or tendency to act in anger and anticipated anxiety and perceive it as a reality. This leads those who suffer from the disorder to behave in a manner characterized by an exaggeration of reacting to others in interpersonal relationships [9]. For our purposes, we defined rejection sensitivity as the total score obtained by students on the scale of rejection sensitivity used in this study.

Social anxiety: a state of observed fear of several social situations, especially performance situations, in which the individual appears in front of unfamiliar people or is under examination or observation from others. In these situations, the individual feels confused, fearful, and embarrassed. The individual realizes that this anxiety is irrational; nevertheless, it affects social activities [1]. For this study, we define social anxiety as "a state of tension resulting from the actual expectation or occurrence of evaluation in imaginary or real social interaction situations in interaction or confrontation situations."

\section{Literature Review}

\subsection{Intervention of Coping Strategies}

Coping resources are components in the environment (e.g., social support) or of an individual (e.g., problem-solving ability), which assist in dealing with stressors. Coping strategies (e.g., engaging in relaxing diversions) are the particular cognitive or behavioral acts used in response to a stressful event. Coping styles (e.g., approach coping) are modes of dealing with stressors that an individual typically engages in as a response to similar stressors over time. There is a multitude of subtypes or categories of coping that are considered either adaptive or maladaptive, such as problem-solving, support seeking, distraction, and self-blame [7]. Passive coping generally involves avoidance, withdrawal, and wishful thinking. And behavioral examples of passive coping strategies include talking to others about the situation either to ventilate feelings, get sympathy or elicit their help, withdrawing from social and other activities.

Williams [35] reports that children and adolescents experience stress in their lives and that they attempt to cope with that stress. Although most research on stress and coping has focused on adults, recent attention to adolescents suggests that there are developmental changes in coping during adolescence and that particular coping strategies vary with gender and the type of stressors adolescents experience. This study examined coping strategies used by male and female students in early, middle, and late adolescence when they were coping with two different types of stressors: daily hassles and major life events. Older adolescents used a greater variety of coping strategies and used methods that directly reduce the impact of the stressor and involved a cognitive component (e.g., plan full problem solving; reappraisal) more often than younger adolescents.

Regarding college students, there are a few studies interested in improving coping strategies (e.g., Dickins [8]). This study found that college students have increased levels of stress, feeling overwhelmed, and the stress has been linked to a variety of physical and emotional problems. A number of studies have identified coping resources as helpful in decreasing the negative effects of stress. However, there are still some questions in the literature regarding effective ways to increase coping resources. Reading written feedback about coping resources is one way to increase individuals' awareness about their coping resources. Another intervention that has been shown to have positive and lasting effects with regard to health and well-being is therapeutic writing. While there are studies that point toward writing being beneficial to coping, there are currently no studies that have specifically connected writing interventions with increased coping strategies. Kadirava \&Kumar [17] reported that stress exists in every part of our lives. The prevalence of stress is increasing among students in recent years. This is due to various factors such as increased pressure, competition, decreased resources, inadequate family support, exposure to violence through media, and increased use of alcohol as well as drugs. The transition from adolescence to adulthood is a difficult journey filled with various risks, rapid changes, and seemingly endless choices. Coping skills can help the youth to navigate through these challenges. These skills can help students to be self-reliant, solve problems, and make informed choices, which in turn promote their physical and psychological well-being.

\subsection{Rejection Sensitivity}

Adolescents with higher rejection sensitivity typically feel insecure and unhappy about their relationships and tend to perceive ambiguous behaviors in significant others as intentional rejection. Marston [25] used longitudinal, multi-reporter data in a community sample to examine the role of rejection sensitivity in late adolescents' social and emotional development. Rejection sensitivity was linked to a relative increase in adolescent depressive and anxiety symptoms. Stephen Ford [32] found that rejection sensitivity is a state of anxious expectation and the perception of other people's actions as a sign of rejection. The model of rejection sensitivity assumes that individuals with previous experiences of rejection become more sensitive to experiences of rejection in the future towards others, and are exposed to misalignment associated with rejection sensitivity. Downey [11] revealed that everyone experiences rejection. But while some people respond with equanimity, others respond in ways that profoundly compromise their well-being and relationships. Research has shown that individuals who anxiously expect rejection have a tendency to readily 
perceive it in other people's behavior and then react to it in ways that undermine their relationships; their behavior thus leads to the feared outcome [4, 9]. McDonald [26] investigated rejection sensitivity, the tendency to anxiously or angrily expect rejection, is associated with internalizing difficulties during childhood and adolescence; Analyses revealed that angry rejection sensitivity was related to depressive symptoms, but only for adolescents reporting low support from parents and friends. Friend support moderated the association between (1) angry rejection sensitivity and social anxiety, and (2) anxious rejection sensitivity and depressive symptoms. For adolescents reporting low support from friends, support from parents was positively related to social anxiety. Findings highlight the importance of considering relationships in studies of rejection sensitivity and adjustment during adolescence.

\subsection{Social Anxiety}

A person with social anxiety may present with various characteristics. For example, he or she may have rigid posture, speak with a quiet voice, display shyness, fail to participate in conversations, and avoid engaging in significant relationships. Kocovski [19] defined social anxiety disorder as the "marked fear of social or performance situations in which one is exposed to unfamiliar people or possible scrutiny by others." Social anxiety is defined in the Diagnostic and Statistical Manual of Mental Disorders (5th ed.; DSM-5; American Psychiatric Association) [3] as a condition characterized by abnormal fears of social situations. Social anxiety indicators can be manifest as physical symptoms (e.g., sweating, headaches, chills), cognitive symptoms (e.g., expecting to perform poorly or engaging in negative self-talk), or behavioral symptoms (e.g., avoidance, withdrawal, or social isolation). Examples of situations feared commonly by individuals with social phobia include participating in meetings, initiating and maintaining conversations, and meeting new people. Rudolph \& Zimmer [31] tested portions of the rejection sensitivity model by investigating associations of parenting and interparental conflict with early adolescents' emotional maladjustment (depressive and social anxiety symptoms) and to identify whether these associations were indirect via their rejection sensitivity and their emotional and behavioral reactions to rejection threat. They found direct associations with elevated symptoms of depression and social anxiety among early adolescents. Understanding these pathways from familial relationships to child and adolescent emotional ill-health allows the development of more nuanced, effective, and multi-stage approaches to intervention.

Previous studies have shown that more than half $(65.4 \%)$ of college students had moderate social anxiety, while nearly a quarter (22.4\%) had severe social anxiety [36].
Previous studies have also revealed that social anxiety not only seriously endangers college students' psychosocial adaptation and interpersonal communication but also their academic achievement. While many students navigate this transitional time successfully, others are confronted by difficulties in both achievement and psychosocial adjustment. One psychosocial factor that may hinder success in university/college is social anxiety, an emotional problem that is often overlooked or hidden from the casual observer [5]. Jefferies \& Ungar [16] reported higher rates of social anxiety symptoms and prevalence. They suggested that levels of social anxiety may be rising among young people and adolescents because they are most at risk. Public health initiatives are needed to raise awareness of social anxiety.

\section{Materials and Methods}

\subsection{Research Sample}

The original community sample consists of all students in King Faisal University. The random sample consists of 60 male students aged 19-22 years, from the College of Education of King Faisal University from the departments of special education, arts education, and physics education. From this group, we selected 20 students with higher levels of rejection sensitivity and social anxiety and low scores on the coping strategies scale as an experimental group. Our research depends on this single sample.

\subsection{Research Instrument}

Coping strategies scale (CSS): this scale, by Toubin [34], consists of 32 items and is divided into 8 subscales consisting of specific coping strategies. These include the following:

Problem Solving: This subscale includes items referring to both behavioral and cognitive strategies designed to eliminate the source of stress by changing the stressful situation.

Cognitive Restructuring: This subscale includes cognitive strategies that alter the meaning of the stressful transaction as it is seen as less threatening, examined for its positive aspects, and viewed from a new perspective.

Social Support: This subscale includes items that refer to seeking emotional support from people in general, one's family, and one's friends.

Express Emotions: This subscale includes items referring to releasing and expressing emotions.

Problem Avoidance: This subscale includes items referring to the denial of problems and the avoidance of thoughts or actions involving stressful events.

Wishful Thinking: This subscale refers to cognitive strategies that reflect an inability or reluctance to reframe or symbolically alter a situation. The items involve hoping 
and wishing that things could be better.

Self-Criticism: This subscale includes items referring to blaming oneself for what has happened. Social Withdrawal: This subscale includes items that reflect blaming oneself for situations and criticizing oneself. Items are rated on a 5 -point scale ranging from 1 (always) to 5 (never). Reliability: by half- split $n=40$ students, person correlation between single and prior items was $(0,75)$, Spearman prawn $(0,90)$, alpha coefficient $(0,67)$, and $\operatorname{Guttman}(0,81)$. Reliability by test-retest after two weeks was $(0,764)$.

Rejection sensitivity Questionnaire (RSQ): by Downey \& Feldman [9], translated into Arabic by the researcher [13], the questionnaire consists of 18 hypothetical situations in which rejection by a significant other is possible (e.g., "You ask a friend to do you a big favor"'). For each situation, participants were first asked to indicate their degree of concern or anxiety about the outcome of the situation (e.g., "How concerned or anxious would you be over whether or not your friend would want to help you out?'”) on a 6-point scale ranging from 1 (very unconcerned) to 6 (very concerned). Participants were then asked to indicate the likelihood that the other person would respond in an accepting manner (e.g., "I would expect that he/she would willingly agree to help me out") on a 6-point scale ranging from 1 (very unlikely) to 6 (very likely). An overall rejection sensitivity score was obtained by weighting the expected likelihood of rejection by the degree of anxiety or concern about the outcome of the request. Many studies (e.g., [9, 11]), have found that internal consistency for each subscale was very high (Cronbach's for Total Rejection Sensitivity, 0.87). In this study, the reliability by test-retest was $(0,82)$ with a statistical coefficient at $(\mathrm{P} \leq 0.01)$.

Social anxiety scale (SAS): by Leary \& Dobbin, translated into Arabic by Abdel-Rahman and Abdel-Maqsoud [1], the scale has 26 items and participants are required to rate their agreement with each item on a 5-point scale. The highest possible score is 130, and higher scores indicate higher levels of social anxiety. Factorial analyses tested the validity of the scale, resulting in the presence of a single factor $74,7 \%$ of variance, and saturated by the values of both dimensions of $0.86-0.85$ respectively. The validity of the test on the social skills scale and of all anxiety interaction, total degree of social anxiety turned out a negative correlation, while the relationship is statistically significant with no statistical coefficient. The correlation coefficient values were $(0,24-$ $0,90-0.34)$ respectively. The reliability consistency correlation was found to be $(0.85-0.87) n=42$ students by internal consistency between to mean domains interaction anxiety and coping anxiety. Coefficient (Spearman prawn) first factor 0.71 , ascend factor, 0.73 , total degree 0.73 . The test-retest reliability after two weeks was 0.77 , which is statistically significant at the $(P \leq 0.01)$. These scores show the high validity and reliability of the scale.

Counseling program: by the researcher, the program aims to improve coping strategies for reducing rejection sensitivity and social anxiety in college students. The program consists of 16 sessions including knowledge and activities focused on problem-solving, cognitive restructuring, expressing emotions, social support, problem avoidance, wishful thinking, self-criticism, and social withdrawal. The program was given to the experimental group in the following sequence:

Weeks 1-3: Students were instructed in and practiced problem-solving skills. During the second and third sessions, they watched a tape on problem-solving steps under the label "Be positive and don't delay today's problem until tomorrow" and practiced the concepts presented under the direction of the researcher.

Weeks 4-5: Students were trained in cognitive reinstructing and disputing irrational beliefs, and taught to engage in more rational beliefs instead. They were told that negative beliefs give rise to negative emotions, so if they changed their negative attitudes about peer relationships, rejection sensitivity, and social anxiety situations the negative feelings associated with them will gradually weaken.

Weeks 6-7: Students were instructed in and practiced the expression of emotions through emotional control. They were taught to avoid anger, even in very exciting situations. Students were instructed in and practiced social support and to provide assistance to others when needed.

Weeks 8-9: Students were instructed in problem avoidance through training to distinguish between effective and ineffective coping styles. They were taught to replace avoidance behavior with positive coping behavior. In addition, they were taught to reduce wishful thinking through training in the importance of making an effort to achieve goals and remember that dreams do not solve problems. They were encouraged to convert aspirations into reality.

Weeks 10-11: These sessions focused on strategies for accepting self-criticism and reducing social withdrawal through training to evaluate themselves in situations of failure and success and situations of social participation with colleagues, such as discussions and opportunities for teamwork. The researcher used some techniques in the program such as:

Discussion and dialogue: This technique shows how individuals respond to what is presented to them by expressing their opinions, emotions, and discussions about the content of sessions. Self-monitoring: This technique makes the individual master and understands coping strategies to reduce rejection sensitivity and social anxiety. When the individual's self-awareness leads to the organization and management of his emotions, and to interact socially with others, then this technique is equivalent to the cognitive technique of 'know yourself' that makes the individual able to identify him- or herself with all its aspects, especially emotional. Social problem 
solving: a strategy in which individuals achieve reasonable solutions. The researcher facilitates this process so that students are able to achieve solutions without help, from focus on behaviors, desires and feelings and not on good or bad behavior. The solution of the problem helps students learn appropriate ways to solve social problems, including when, how, and where. In addition, possible steps may include identifying the problem, identifying solutions, selecting alternatives, predicting results, and then evaluating them. All sessions included group discussion, short lectures, stories, feedback, reinforcement, modeling, role-play, and homework were used in the sessions might be effective in enhancing coping strategies.

\section{Results}

Table 1. Mean, standard deviation, and t-test on dimensions of coping strategies between pretest and posttest. The results showed that there was a statistically significant difference at 0.01 level between pretest and post-test results, in favor of post-test, in dimensions of problem-solving, cognitive restructuring, expressing emotions, social support, problem avoidance, wishful thinking, self-criticism, and social withdrawal.

Table 2. Mann-Whitney test on differences in rejection sensitivity and social anxiety between pretest and posttest. The results showed that there is a statistically significant difference at 0.01 level between pretest and post-test in total degree of rejection sensitivity and social anxiety in favor of post-testing.

Table 1. Mean, standard deviation and T- test on dimensions of coping strategies between pre-posttest.

\begin{tabular}{|c|c|c|c|c|}
\hline Subscale & Pre/posttest & Mean & S.D & $\begin{array}{c}\text { 'T'- } \\
\text { value }\end{array}$ \\
\hline \multirow{2}{*}{$\begin{array}{c}\text { Problem } \\
\text { solving }\end{array}$} & pre & 49.20 & 2.57 & \multirow{2}{*}{$21.45 * *$} \\
\cline { 2 - 4 } & post & 66.10 & 2.51 & \\
\hline $\begin{array}{c}\text { Cognitive } \\
\text { restructuring }\end{array}$ & pre & 50.30 & 2.32 & \multirow{2}{*}{$23.99 * *$} \\
\cline { 2 - 4 } & post & 67.50 & 2.16 & \\
\hline \multirow{2}{*}{$\begin{array}{c}\text { Express } \\
\text { emotions }\end{array}$} & pre & 50.75 & 3.88 & \multirow{2}{*}{$12.33^{* *}$} \\
\cline { 2 - 4 } Social support & post & 62.65 & 2.36 & \\
\cline { 2 - 4 } & pre & 51.15 & 4.59 & \multirow{2}{*}{$12.22^{* *}$} \\
\hline \multirow{2}{*}{$\begin{array}{c}\text { Problem } \\
\text { avoidance }\end{array}$} & pre & 64.50 & 2.37 & \\
\cline { 2 - 4 } & post & 48.68 & 2.36 & \multirow{2}{*}{$23.17 * *$} \\
\hline \multirow{2}{*}{$\begin{array}{c}\text { Wishful } \\
\text { thinking }\end{array}$} & pre & 66.04 & 2.40 & \multirow{2}{*}{$21.08 * *$} \\
\cline { 2 - 4 } & post & 49.59 & 2.79 & \\
\hline \multirow{2}{*}{ Self -criticism } & pre & 61.90 & 3.59 & \multirow{2}{*}{$12.23 * *$} \\
\cline { 2 - 4 } & post & 50.05 & 3.94 & \\
\hline \multirow{2}{*}{$\begin{array}{c}\text { Social } \\
\text { withdrawal }\end{array}$} & pre & 68.20 & 2.48 & \multirow{2}{*}{$25.17 * *$} \\
\cline { 2 - 4 } & post & 51.25 & 3.39 & \\
\hline
\end{tabular}

Note. ${ }^{*} \mathrm{p}<.05 . * * \mathrm{p}<.01$.
Table 2. Man-Whitney test on rejection sensitivity, and social anxiety between pre-posttest

\begin{tabular}{|c|c|c|c|c|c|}
\hline Factor & \multicolumn{2}{|c|}{ pretest } & \multicolumn{2}{c|}{ posttest } & z- value \\
\hline- & mean & sum & mean & sum & \\
\hline $\begin{array}{c}\text { Rejection } \\
\text { sensitivity }\end{array}$ & 30.33 & 605 & 10.63 & 210 & $5.332^{* *}$ \\
\hline social anxiety & 3.45 & 609 & 10.35 & 208 & $5.420^{* *}$ \\
\hline
\end{tabular}

Note. ${ }^{*} \mathrm{p}<.05 .{ }^{* *} \mathrm{p}<.01$.

\section{Discussion}

Previous research has $[10,18,26,31]$ demonstrated that rejection sensitivity is related to social anxiety and other adjustment difficulties, because some of the psychological difficulties are associated with rejection sensitivity among college students. Thus, programs that involve activities and peer participation may be beneficial in reducing rejection sensitivity and social anxiety. We found significant differences in proactive and non-proactive coping in our experimental group due to our intervention program. The sixteen-session program, including group discussion, role-playing, modeling, stories, and so on, might be effective in enhancing coping skills and reducing rejection sensitivity and social anxiety. Many college students use undesirable coping strategies such as withdrawal and avoiding problems, whereas others' use of coping skills such as problem-solving, cognitive restructuring, and expressing emotions may prompt better adaptation with peers in higher education.

Coping skills can help students be resilient, solve problems, and have a higher sense of autonomy and relatedness in peer relationships that in turn lead to improved psychological health. Students who possess proactive coping strategies for life events, challenges, and stress are likely to be more adjusted in their psychological and social lives. Hence, reducing rejection sensitivity and social anxiety in students, especially at the college level, is essential to proactive behavior.

The findings revealed that the students who participated in the coping strategies counseling program differed significantly between pretest and posttest results in productive coping, and showed improvement in unproductive coping (e.g., problem avoidance, wishful thinking, and social withdrawal). These results agree with previous studies (e.g., $[6,8,7,17])$ on programs designed to help participants improve coping strategies, especially those that focus on problems or emotions. The experimental group reported an increase in the use of adaptive coping strategies and a decrease in the use of maladaptive coping strategies.

Our sessions helped participants learn to work in groups, which enhanced social interaction. We also gave students training in coping strategies, which helped them in facing problems and seeking appropriate solutions. The program included many activities aimed at improving coping 
strategies and using problem-solving strategies to reduce levels of social rejection and social anxiety. The problem-solving method is situated between fully aware of previous information, and not fully aware of the position of a new exhibit and the information and skills can be used in solve the problems, and to organize his experience and his knowledge before choosing from that applied in the new problem.

Cognitive restricting, which indicate to all treatment models about modified cognitive factors, may be due to the maladjustment behavior that resulting from irrational thinking, reorganized the perceptual field, rearrange the ideas, and understanding the negative effect to some thinking styles, and make the necessary exchange the maladjustment thinking styles to adjustment, affective, wisdom, and self-control. In addition, counseling helped participants learn how to control the expression of anger and frustration in appropriate ways in virtual and real situations.

The current program has helped participants reduce rejection sensitivity through training in strategies, tasks, exercises, and activities. It has also helped them to develop potential and to learn the strengths and weaknesses in themselves. Consequently, they can strengthen weak areas, and achieve their goals by taking advantage of potential and abilities of which they were previously unaware. The program sessions required students to participate in collective work and social interactions. It also helped the experimental group take advantage of the rejection sensitivity strategies in which they had been trained, which will help them in dealing with peers and in making friendships, awareness, and different solutions of some problems through the realistic situation by role-playing, scenario, and modeling.

Social anxiety is a particularly important factor during the transition from high school to university as it influences the development of new relationships. Thus, our program has helped students reduce social anxiety through sessions that included interaction between peers, expressing emotions, facing problems, and learning not to avoid or withdraw from situations. The program has increased students' self-confidence in communicating and interacting with others and developed students' abilities to turn negative strategies such as avoiding problems, wishful thinking, self-criticism, and social withdrawal into positive ones such as cognitive restructuring, expressing emotions, and social support.

Thomason [33] reported that self-efficacy for individuals contributes to social anxiety and the perception and awareness of individuals in their ability to cope. For example, some individuals may adopt learned helplessness. Students with high levels of social anxiety are more likely to find a social situation threatening and are less likely to perceive themselves as having control in the situation [19].

Social anxiety is one of the most common problems that break down integration with others in the community, but we can overcome this problem by developing social skills that can help students with social interaction. Thus, reducing social anxiety can be achieved through involvement in various activities collective tasks. Many studies have found that individuals with social anxiety disorder show a weakness in understanding coping strategies and use avoidance in dealing with pressing social situations. Their use of coping based on emotion, especially avoidance and withdrawal, indicates the presence of internal problems.

\section{Conclusions}

Based on the results of the research and discussion that has been described, we conclude that proactive coping strategies can help students reduce rejection sensitivity and social anxiety by enhancing their coping strategies through training programs. Students became more proactive which in turn prevented a sense of rejection sensitivity and social anxiety. Previous studies had demonstrated the role that counseling programs play in decreasing adolescents' rejection sensitivity and social anxiety. Despite that, these counseling programs have not received much attention. The current study found that improvement of coping strategies reduces the degree of rejection sensitivity and social anxiety. The results showed significant differences in coping strategies: problem-solving, cognitive restructuring, emotions expressing, social support, problem avoidance, wishful thinking, self-criticism, and social withdrawal in favor of post-test data. We also found significant differences in rejection sensitivity and social anxiety in favor of counseling program post-testing. Many college students need to acquire coping strategies for rejection sensitivity and social anxiety. Reducing these disorders is an important factor in achieving mental health and getting on a path to success, confidence, and increased quality of life. In sum, teaching effective coping skills is a worthwhile avenue for intervention and prevention.

\section{Recommendations}

In light of our findings, we recommend more training and counseling programs to enhance coping strategies to reduce rejection sensitivity and social anxiety. We recommend additional research in light of variables that are not addressed in the current study, such as resilience, optimistic thinking, emotional regulation, spiritual intelligence, and effective parental. Also, there should be experimental studies aimed at designing counseling programs to improve coping strategies for reducing rejection sensitivity and social anxiety. Psychological and professional counselors should prepare training programs on proactive coping strategies such as problem-solving, cognitive restructuring, expressing emotions, social 
support, and improvement of unproductive coping (e.g., problem avoidance, wishful thinking, self-criticism, and social withdrawal). In addition, educators and developers of higher-education curricula can include activities to activate coping strategies in college courses. Attention should be paid to designing active learning environments and providing opportunities for students to participate and interact with each other to avoid rejection sensitivity and social anxiety. This is especially vital for college students in education departments because they will be the teachers of the future.

\section{Acknowledgements}

The author acknowledges the Deanship of Scientific Research at King Faisal University for financial support, under Track annual research No. (160132).

\section{REFERENCES}

[1] Abdel-Rahman, M. El-Sayed, and Abdel-Maqsoud, Hanim (1998). Social skills, affirmative behavior, social anxiety and their relationship to helping others among university students. In: Abdel-Rahman: Studies in mental health. Part 2, pp. 149-199, Cairo, Qubaa Printing and Publishing.

[2] Al-Hazmi BH, Sabur SS, Al-Hazmi RH. (2020). Social anxiety disorder in medical students at Taibah University, Saudi Arabia. J Family Med Prim Care; 9:4329-32.

[3] American Psychiatric Association. (2013). Diagnostic and statistical manual of mental disorders (5th ed.). Washington, DC: Author.

[4] Ayduk, O; Downey G; Kim M. (2001). Rejection sensitivity and depressive symptom in women. Personality\& Social psychology Bulltin, 21 (7), 868-877. doi: 10.1177/ 0146167201277009.

[5] Brook, C. A., \& Willoughby, T. (2015). The social ties that bind: Social anxiety and academic achievement across the university years. Journal of Youth and Adolescence, 44(5), $1-14$.

[6] Bugalski, K.; Frydenberg, E (2000). 'At Promoting effective coping in adolescents risk' for depression. Australian Journal of Guidance and Counseling, 10 (1), 111-132.

[7] Carter Alina Erin, (2010). Evaluating the Best of Coping Program: Enhancing Coping Skills in Adolescents. Degree of Doctor, University of Windsor, Faculty of Graduate Studies, Ontario, Canada.

[8] Dickinson Wendy L. (2006). Increasing coping resources: An experimental intervention approach. Degree of Doctor. The College of Education, Georgia State University Atlanta.

[9] Downey, G., \& Feldman, S. I. (1996). Implications of rejection sensitivity for intimate relationships. Journal of personality and Social Psychology, 70, 1327-1343. doi:10.1037/0022-3514.70.6.1327.
[10] Downey, G., Lebolt, A., Rincón, C., \& Freitas, A. L. (1998). Rejection sensitivity and children's interpersonal difficulties. Child Development, 69, 1074-1091. doi:10.1111/j.1467-86 24.1998. tb06161.x.

[11]Downey, G. Mougios, V. Ayduk, O. London, B.(2004). Rejection sensitivity and the defensive motivational system: Insights from the startle response to rejection cues. Psychological Science, 15,668-673.

[12] Downey, G. Feldman, S. \&.Ayduk, O.(2000). Rejection sensitivity and male violence in romantic relationships. Personal relationship, 7 (1), 45-61.

[13] Elballah A. Khaled (2013). The quality of friendship and its relationship to depressive symptoms and rejection sensitivity among university students. Journal of the College of Arts, Menoufia University. Number 92, January pp. 679-734.

[14] Hakami RM; Mahfouz MS; Adawi AM; Mahha AJ; Athathi AJ; \& Daghreeri HH, (2017). Social anxiety disorder and its impact in undergraduate students at Jazan University, Saudi Arabia. Ment Illn; 9:7274.

[15] Inguglia Cristiano, Costa Sebastiano Ingoglia, Sonia, Cuzzocrea Francesca \& Liga Francesca (2020). The role of parental control and coping strategies on adolescents' problem behaviors. Current Psychology https://doi.org/10.1 007/s12144-020-00648-w

[16] Jefferies P, Ungar M (2020) Social anxiety in young people: A prevalence study in seven countries. PLoS ONE 15 (9),1-18 DOI:10.1371/journal.pone.0239133

[17] Kadhiravan. S., \& Kumar, K. (2012). Enhancing stress coping skills among college students. International Refereed Research Journal. Vol.3, Issue-4(1), 49-55. www.researche rsworld.com

[18] Kelliher, Jessica Leigh (2013). Personality, rejection sensitivity and perceptions of social support adequacy as predictors of college students, depressive symptoms. Degree of master, faculty of the graduate school of western Carolina University.

[19] Kocovski, Nancy (2001). Attentional biases and coping with social anxiety. Degree of Doctor, Faculty of graduate studies, York University, Toronto, Ontario.

[20] Khoshkam S, Bahrami F, Ahmedi A, Fatehizade M, Etemadi O (2012) Attachment style and rejection sensitivity: The mediating effect of self-esteem and worry among Iranian college students. Eur J Psychol, 8:363-374.

[21]Lazarus, R.S., \&Folkman, S. (1984). Stress appraisal and coping. New York: Springer.

[22] Lewis, R., \& Frydenberg, E. (2004). Students' self-evaluations of their coping: how well do they do it? In Frydenberg, E. (Ed.), Thriving, surviving, or going under: coping with everyday lives. Greenwich, CT: Information Age Publ. Pp. 25-44.

[23] Lopze, Shane J. (2013). The encyclopedia of positive psychology. Wiley- Blackwell.

[24]London, B., Downey, G., Bonica, C., \& Paltin, I. (2007). Social causes and consequences of rejection sensitivity. Journal of Research on Adolescence, 17, 481-506. doi:10.1111/j.1532-7795.2007.00531.x. 
910 Effectiveness of Coping Strategies Program in Reducing the Rejection Sensitivity and Social Anxiety with College Students

[25] Marston, Emily, G. Amanda, Hare, \& Joseph, P. Allen.(2010).Rejection sensitivity in late adolescence: Social emotional sequela. The Research Adolescence, 20 (4), 959-982.

[26] McDonald Kristina; bowker Julie; Rubin Kenneth; brett laursen\& duchene Melissa (2010). Interactions between rejection sensitivity and supportive relationships in the prediction of adolescents' internalizing difficulties. Journal of youth adolescence, 39:563-574.

[27] Morrison, A. S., \& Heimberg, R. G. (2013). Social anxiety and social anxiety disorder. Annual Review of Clinical Psychology, 9(1), 249-274.

[28] Özen A, Sümer, Demir M (2010). Predicting friendship quality with rejection sensitivity and attachment security. J Soc Pers Relat, 28:163-181.

[29] Pittman Laura \& Richard Adeya (2008). University belonging friendship quality and psychological adjustment during transation to college. The Journal of Excremental Education, 76 (4), 343-361.

[30] Richardson Cele E., Magson Natasha, Fardouly Jasmine, Oar Ella, Forbes Miriam, Johnco1 Carly \& Rapee Ronald M. (2020). Longitudinal Associations between Coping Strategies and Psychopathology in Pre-adolescence. Journal of Youth and Adolescence. https://doi.org/10.1007/s10964-0 20-01330-x

[31] Rudolph Julia \& Zimmer-Gembeck, Melanie. (2014). Parent relationships and adolescents' depression and social anxiety: Indirect associations via emotional sensitivity to rejection threat. Australian Journal of Psychology, 66: 110-121 doi: 10.1111/ajpy. 12042

[32] Stephen, Ford (2012). Appearance - based sensitivity among college freshmen. Degree of Doctoral, Walden University.

[33] Thomasson, P. \& Psouni, E. (2010). Social anxiety and related social impairment are linked to self-efficacy and dysfunctional coping. Scandinavian Journal of Psychology, $51,171-178$.

[34] Tobin, David. (2001). User manual for the coping strategies inventory. https://www.academia.edu/30133319/User_Manual_for_the COPING_STRATEGIES_INVENTORY. Cited on 14 Nove 2020

[35] Williams Kristin \& Ann McGillicuddy -De Lisi (2000). Coping strategies in adolescents. Journal of applied Developmental Psychology, 20 (4), 537-549.

[36] Wu, X., Huang, L., He, X., Tang, H., \& Pu, W. (2015). Social anxiety, aggression and depression: The mediating of regulatory emotional self-efficacy. Chinese Journal of Clinical Psychology, 23(5), 804-807 [in Chinese].

[37] Yi Yu; China Shen; Liu Hefei; Hang Fan, \& Lin Zhang (2020). Effect of Parent-Child Attachment on College Students' Social Anxiety: A Moderated Mediation Model. Psychological Reports, 123 (6), 2196-2214. DOI: $10.1177 / 0033294119862981$

[38]Zhao, C., \& Dai, B. (2016). Relationship of fear of negative evaluation and social anxiety in college students. China Journal of Health Psychology, 24 (11), 1746-1749. 